\title{
Historische Vergleichung als Erkenntnismethode. Die vergleichende Beobachtung von Recht und Staat im 18. Jahrhundert
}

\author{
Heinz Mohnhaupt
}

Max-Planck Institut für Rechtsgeschichte und Rechtstheorie Kontaktnie-mail:mohnhaupt@rg.mpg.de

Historical Comparison as Method of Cognition. The Comparative Observation of Law and State in 18th Century

\begin{abstract}
:
The comparison and comparation (also comparatio, comparative studies) present today a general method of cognition for determining similarities and differences in society. Most sciences (law, anatomy, politics, languages, culture etc.) have their own comparative foundations, although these form separate disciplines or methods. Comparative law has been developing as a science since the 19th century. The historical background and discussion about the purpose and benefits of comparison are discussed in this article. They originate mostly in the universalism of the Enlightenment philosophy, the practical approach of which is based on comparative methodological principles, which it uses for legal development of the state and society. In the 18th century, this mainly involved determination of the "interest" of the state and creation of a systematic codification.
\end{abstract}

Keywords: comparison; comparative methods; comparative law

DOI: $10.14712 / 2464689 X .2021 .3$

\section{Historische Vergleichung in Theorie und Praxis der Wissenschaften als Erkenntnisverfahren mit offener Methodik}

Die Aufgabe, über Vergleichung - oder besser über das Vergleichen - zu sprechen, hat zwei Aspekte zu bedenken. Zum einen handelt es sich um einen banalen Vorgang der Beobachtung bzw. des Beobachtens von unterschiedlichen Handlungen, Ereignissen oder Personen, die wir selber täglich bewusst oder unbewusst vornehmen. Wir messen z.B. 
eigenes Verhalten am Verhalten anderer, stellen Eigenschaften von Personen oder Institutionen einander gegenüber oder suchen Ähnlichkeiten in unterschiedlichen Ereignissen. Diese elementaren gedanklichen oder visuellen Operationen bezwecken und ermöglichen Bewertungen, Orientierungen, bilden Maßstäbe und Positionen, aus denen Ursachen für die beobachteten Phänomene gefunden werden, die oft mit Wiedererkennungseffekten verbunden sind. Solche „elementaren“ Beobachtungen und Operationen können ins „Uferlo$\mathrm{se}^{\text {"1 }}$ führen und bilden so eine unreflektierte „Allerweltsoperation“. ${ }^{2}$ Alles kann verglichen werden, um Positionsbestimmungen zu finden und zu begründen. 1767 erklärte ein anonymer Autor über den oft beklagten „unglücklichen“ Zustand des „Heiligen Römischen Reichs deutscher Nation“: „Sind wir aber denn in der That so unglücklich ... Ich glaube nicht. Das ist ausgemacht: daß alles Glück und Unglück nur auf unserer Einbildungskraft beruhet; ... Daß diese unsere Einbildung aus der Vergleichung mit anderen entstehe. "3

Als Länder, die er zum Vergleich heranzieht, benennt er ausdrücklich: Polen, Schweden, Russland, Dänemark, Frankreich, Spanien, die Vereinigten Niederlande, England, die Schweiz, ohne allerdings in eine konkrete Vergleichung einzutreten. Das behauptete „Glück“ betraf die Regierungsverfassung des Alten Reiches, die hier deren allgemeinen gesellschaftlichen und rechtlichen Zustand bedeutet. ${ }^{4}$ Dieser wird am Zustand der anderen europäischen Staaten gemessen. Der anonyme Autor erklärt weiter: „Unser Glück beruhet auf unserer Einbildungskraft und diese bestehet auf der Vergleichung mit andern. Vergleiche ich Deutschlands Unterthanen mit andern: so kann es sein, daß ich ihr Unglück für gering halte." 5

Diese „Einbildung“ - wie sie der Autor nennt - ist eine Bewusstseinsbildung, die auf Vergleichung beruht. Fast das ganze Europa wird vom beobachtenden Autor zur vergleichenden „Glücks“-Bewertung herangezogen. Denken wir an andere Kulturphänomene wie Mentalitäten und nationale Eigenschaften, so resultieren z.B. Charakterisierungen deutscher Nationaleigenschaften, wie man sie bei Germaine Staël, Mark Twain oder Stendhal lesen kann, aus einer vergleichenden Beobachtung und Wahrnehmung. ${ }^{6}$ Louis Basset de la Marelle hat z.B. in Frankreich den Vergleich auf Nationalcharakter und Patriotismus bezogen, um deren Differenz zu England, Italien, Dänemark, Deutschland und dem alten Rom zu ermitteln. ${ }^{7}$ Der Vergleich mit anderen Völkern ergänzt und schärft das Bild der Beobachter von sich selbst wie auch der Beobachteten. Das gilt in gleicher Weise für das sich vergleichende und verglichene Individuum. Selbsterkenntnis und Selbstvergewisserung

1 Cf. BICHLER, R. Die theoretische Einschätzung des Vergleichens in der Geschichtswissenschaft. In: HAMPEL, F. - WEILER, I. (hrsg.). Vergleichende Geschichtswissenschaft. Methoden, Resultate und ihr Beitrag zur Universalgeschichte. Darmstadt: Wissenschaftliche Buchgesellschaft, [Abt. Verl.], 1978, S. 1.

2 So MIDDELL, M. Kulturtransfer und Historische Komparatistik - Thesen zu ihrem Verhältnis. Comparativ. Leipziger Beiträge zur Universalgeschichte und vergleichenden Gesellschaftsforschung, 2000, 10, S. 10.

3 Freie Gedanken über einige der neuesten Staats-Strittigkeiten. Geschrieben im H. R. Reich Deutscher Nation, s.l. 1767, S. 35 (Hervorhebung von H. M.).

4 Dazu MOHNHAUPT, H. Verfassung I. In: GRIMM, D. - MOHNHAUPT, H. Verfassung. Zur Geschichte des Begriffs von der Antike bis zur Gegenwart. Zwei Studien (Schriften zur Verfassungsgeschichte 47). 2. Aufl. Berlin: Duncker \& Humblot GmbH, 2002, S. 71-75.

$5 \quad$ Freie Gedanken, S. 97

6 DEMANDT, A. Über die Deutschen. Eine kleine Kulturgeschichte. Berlin: Propyläen Verlag, 2007, S. 471.

7 BASSET DE LA MARELLE, L. La difference du patriotisme national chez les François et chez les Anglois. Paris, 1766. 
beruhen auf Vergleichung, wie Goethe im „Torquato Tasso“ Antonio sagen lässt: „Und wenn Du ganz dich zu verlieren scheinst, Vergleiche Dich! Erkenne, was Du bist.“ Worauf Tasso antwortet: „Damit ich mich mit ihm vergleichend fasse."8 Mit ähnlichen Worten beschrieb Thomas Mann dieses Vergleichsziel: „Denn nur durch Vergleichung unterscheidet man sich und erfährt, was man ist, um ganz zu werden, was man sein soll. "9 Man kann dieses allgemeine, strukturierende Beobachtungs- und Denkprinzip auch das „Verstehen durch Vergleichen“ nennen. ${ }^{10}$ Novalis formulierte: „Alles Erkennen kommt aus dem Vergleichen. "11 Das gilt sowohl für die psychische Welt des Fremdartigen und Fremden ${ }^{12}$ als auch für die realen gesellschaftlichen Institutionen und rechtlichen Organisationsformen staatlicher Gemeinwesen.

Der zweite Aspekt meiner Ausführungen betrifft die Frage des Umschlags dieses elementaren - sei es bewussten oder unbewussten - vergleichenden Beobachtens in ein reflektiertes Verfahren mit dem Anspruch auf eine wissenschaftlich fundierte Erkenntnismethode. Damit ist deren Nachprüfbarkeit auf Grund eines Methodenkonsenses und einer kritischen Kommunikation über die vergleichenden Arbeiten und deren Ergebnisse gemeint. ${ }^{13}$ Die Frage, inwieweit Vergleichung diesem wissenschaftlichen Anspruch genügt, betrifft grundsätzlich alle wissenschaftlichen Disziplinen von der Medizin, Geschichte, Religion usw. bis zum Recht, soweit diese vergleichend arbeiten. Überblickt man die einzelnen wissenschaftlichen Disziplinen, so ergibt sich heute kein einheitliches methodisches Verfahrensbild, obwohl alle Wissenschaften die Vergleichung als Erkenntnismittel fordern und überwiegend auch praktizieren. ${ }^{14}$ Diese Forderung ist Ausdruck einer Grenzen

8 GOETHE, J. W. Torquato Tasso, Fünfter Auftritt. In: BEUTLER, E. (hrsg.). Gedenkausgabe. 6. Band. Zürich: Artemis Verlag, 1954, S. 313; ähnlich GOETHE, J. W. „Briefe aus der Schweiz“. In: BEUTLER, E. (hrsg.). Gedenkausgabe. 9. Band. Zürich, Artemis Verlag, 1954, S. 486: „... ja ich habe die römische Geschichte gelesen, um bei der Vergleichung recht lebhaft zu fühlen, was für ein armseliger Schlucker ich bin."

9 Th. Mann, Gesammelte Werke. Band 5. 2. Aufl. Frankfurt a. M.: S. Fischer, 1974, S. 142; hier zitiert nach RÖSLER, H. Rechtsvergleichung als Erkenntnisinstrument in Wissenschaft, Praxis und Ausbildung. Jus, 1999, S. 1086.

10 Cf. PATSCH, H. Verstehen durch Vergleichen: Die Biblia Pentapla von 1710-1712. In: BEETZ, B. CACCIATORE, G. (hrsg.). Die Hermeneutik im Zeitalter der Aufklärung. Köln (et al.): Böhlau, 2000, S. 113.

11 So das Motto bei ZWEIGERT, K. Nationale Rechtstraditionen, Weltrecht und Gerechtigkeit. In: CLAUSSEN, C. P. (hrsg.). Neue Perspektiven aus Wirtschaft und Recht. Festschrift für Hans Schäffer zum 80. Geburtstag. Berlin: Duncker \& Humblot Gmbh, 1966, S. 333-342.

12 Cf. in diesem Zusammenhang Goethes bekannten Ausspruch: „Wer fremde Sprachen nicht kennt, weiß nichts von seiner eigenen.“ In: BEUTLER, Gedenkausgabe. 9. Band, S. 508; ähnlich S. 632: „Beim Übersetzen muß man bis ans Unübersetzliche herangehen; alsdann wird man aber erst die fremde Nation und die fremde Sprache gewahr."

$13 \mathrm{Zu}$ Kriterien und Anforderungen an disziplinäre Verselbständigung wissenschaftlicher Fragestellungen cf. SCHRÖDER, W. Disziplingeschichte als wissenschaftliche Selbstreflexion der historischen Wissenschaftsforschung. Frankfurt am Main - Bern: Peter Lang, 1982, S. 157; MOHNHAUPT, H. Vorstufen der Wissenschaften von „Verwaltung“ und ,Verwaltungsrecht“ an der Universität Göttingen (1750-1830). In: HEYEN, E. V. (hrsg.). Formation und Transformation des Verwaltungswissens in Frankreich und Deutschland (18./19.Jh). Baden-Baden: Nomos Verlagsgesellschaft, 1989, S. 75 ss. (n. 16).

14 So legt auch die Satzung des „Max-Planck-Instituts für europäische Rechtsgeschichte“ vom 5. Juni 2003 in $\S 1$ Abs. 2 fest: „,.. Die vergleichende Erforschung der Rechtsgeschichte der europäischen Länder soll ihre Gemeinsamkeiten und Divergenzen der Rechtstraditionen aufzeigen. Zugleich soll damit ein Beitrag zur Rechts- und Gesellschaftstheorie erbracht werden.“ 
überschreitenden Universalisierung, die territoriale, nationale und disziplinäre Begrenzungen zu überwinden sucht. Das ist sowohl in der Geschichte der Geschichtswissenschaft als auch in der des Rechts zu beobachten. In seiner docta ignorantia - dem Traktat über die „,belehrte Unwissenheit“ - hat Nicolaus von Cues die Vergleichung 1448 als ein allgemein formuliertes wissenschaftliches Gebot formuliert: Omnes autem investigantes in comparatione praesuppositi certi proportionabiliter incertum iudicant. Comparativa est omnis inquisitio medio proportionis utens... Omnis igitur inquisitio in comparativa proportione facili vel difficili exsistit. ${ }^{15}$

Die Verwissenschaftlichung des Vergleichens als ein methodisches Prinzip setzt jedoch erst im 19. Jahrhundert ein. Friedrich Nietzsche nannte dieses „Zeitalter der Culturen“ in einem Aphorismus das ,Zeitalter der Vergleichung“: „Ein solches Zeitalter bekommt seine Bedeutung dadurch, dass in ihm die verschiedenen Weltbetrachtungen, Sitten, Culturen verglichen und neben einander durchlebt werden können; ... Es ist das Zeitalter der Vergleichung. "16

Was Nietzsche 1878 als allgemeinen Kulturvergleich bezeichnet hatte, besaß in Veröffentlichungen von Albert Hermann Post ${ }^{17}$ und Emerico Amari ${ }^{18}$ einen deutlichen Bezug zu Rechtswissenschaft und Gesetzgebung auf vergleichender Grundlage. Erik Jayme hat erkennbar gemacht, dass beide Autoren mit ihren Vergleichsintentionen Nietzsche bekannt gewesen sind. ${ }^{19}$ Als ein Vertreter dieses Zeitgeistes erklärte auch Gustav Droysen ab 1858 in seinen Vorlesungen über „Enzyklopädie und Methodologie“ in der Geschichtswissenschaft die analogische Betrachtung als Vergleichung: „Die Analogie zwischen zwei X, soweit sie sich gegenseitig ergänzen, wird zum komparativen Verfahren. “20

Zumeist besteht das Untersuchungsziel darin, durch Kontrastierung die Unterschiede in den Vergleichsfällen herauszuarbeiten oder die Gemeinsamkeiten zu ermitteln, die wiederum auf Beziehungen und Zusammenhänge zwischen den Vergleichstatbeständen schließen lassen. ${ }^{21}$ In diesem Sinne formulierte Otto Hintze 1929 zwei Erkenntnisziele der

15 DE CUSA, N. De docta ignorantia. Die belehrte Unwissenheit. Liber I. Ed. und übersetzt von P. Wilpert. 2. ed. von H. G. Senger. Hamburg: Verlag Von Felix Meiner, 1967, S. 6 (deutsch: “Alle Forscher urteilen über etwas Ungewisses, indem sie diese Ungewissheit bezüglich ihres proportionalen Verhältnisses zu einer vorausgesetzten Gewissheit in Vergleichung stellen. Vergleichend verfährt also jede Forschung, indem sie das Mittel der Verhältnisbestimmung anwendet... Jede Forschung besteht also in Vergleichungen und Beziehungssetzungen, mögen diese auch manchmal einfacher oder schwieriger sein.“

16 NIETZSCHE, F. W. Zeitalter der Vergleichung. In: Menschliches, Allzumenschliches. Ein Buch für freie Geister (23. Aphorismus). Band I. In: COLLI, G. - MONTINARI, M. (hrsg.). Sämtliche Werke. Kritische Studienausgabe. Band II. München: Deutscher Taschenbuch Verlag, 1980, S. 44.

17 POST, A. H. Bausteine für eine allgemeine Rechtswissenschaft auf vergleichend-ethnologischer Basis I-II. Oldenburg, 1880-1881; cf. zu Post vor allem KIESOW, R. M. Das Naturgesetz des Rechts. Frankfurt am Main: Suhrkamp, 1997, S. 36 ss.

18 AMARI, E. Critica di una scienza delle legislazione comparate. Genova, 1857.

19 JAYME, E. Das Zeitalter der Vergleichung - Emerico Amari (1810-1870) und Friedrich Nietzsche (1844-1900). In: MAZZACANE, A. - SCHULZE, R. (hrsg.). Die deutsche und die italienische Rechtskultur im „Zeitalter der Vergleichung “. Berlin: Duncker \& Humblot, 1995, S. 21-29 (21-23).

20 DROYSEN, J. G. Historik. Vorlesungen über Enzyklopädie und Methodologie der Geschichte. Im Auftrag der Preussischen Akademie der Wissenschaften herausgegeben von Rudolf Hübner. 2. Aufl. München und Berlin: Verlag von R. Oldenbourg, 1943, S. 340 (§ 39: Die pragmatische Interpretation).

21 Cf. HAUPT, H.-G. - KOCKA, J. Historischer Vergleich: Methoden, Aufgaben, Probleme. Eine Einleitung. In: DIESELBEN (hrsg.). Geschichte und Vergleich. Ansätze und Ergebnisse international vergleichender Geschichtsschreibung. Frankfurt am Main: Campus Verlag, 1996, S. 11. 
Vergleichung auf der Grundlage von Abstraktion und Typenbildung: „Alle Typenbildung ist anschauliche Abstraktion und beruht auch schon auf einem vergleichenden Verfahren. ... man kann vergleichen, um ein Allgemeines zu finden, das dem Verglichenen zugrunde liegt; und man kann vergleichen, um den einen der verglichenen Gegenstände in seiner Individualität schärfer zu erfassen und von dem andern abzuheben. Das erstere tut der Soziologe, das zweite der Historiker." 22

Als Historiker ging es Hintze darum, die „,vergleichende(n) historische(n) Betrachtung des Staatslebens der neueren Völker in einen systematischen Zusammenhang“ zu stellen, um auf diese Weise einen Beitrag zur „Staatswissenschaft“ zu leisten, ${ }^{23}$ d.h. die Entwicklung unterschiedlicher Verfassungsformen zu erkennen. Das zeitliche Untersuchungsfeld der Vergleichung reichte bei Hintze vom Spätmittelalter bis zum Ende des 19. Jahrhunderts. Auch Marc Bloch, der natürlich in diese Reihe der Stimmen über die Methode der Vergleichung gehört, ging vom Mittelalter aus. In seinem berühmten Plädoyer von 1928 hatte er „pour une histoire comparée des societés européennes“ geworben. ${ }^{24}$ Er begreift die „méthode comparative“ auch als Teil der „,philosophie de l'histoire ou de la sociologie générale“ und geht ähnlich wie Droysen von Analogien aus: „... présenter ... certaines analogies, décrire ... leurs évolutions, constater les ressemblances et les différences et ... expliquer les unes et les autres." 25

Dieser so beschriebene Vergleichszweck entspricht auch heute noch den gängigen Definitionen komparatistischer Verfahren. ${ }^{26}$ Freilich warnt Bloch auch davor, in der Vergleichung eine „panacée nouvelle“ zu sehen: „La methode comparative peut beaucoup ... Mais elle ne peut pas tout: en science, pas de talisman.“27

Für Bloch bedeutet diese Methode „un instrument technique“ mit Nutzen ,à l'histoire des institutions politiques, économiques, et juridiques ...".28 Dieses ,instrument technique" hat in den modernen historisch arbeitenden Wissenschaften auch Kritik und Zweifel an seiner Effektivität erfahren. ${ }^{29}$ Für die Geschichtswissenschaft hat Michel Espagne den Einwand erhoben, dass der Vergleich im Grunde tautologisch sei und nur beweisen

22 HINTZE, O. Soziologische und geschichtliche Staatsauffassung. Zu Franz Oppenheimers System der Soziologie. In: OESTREICH, G. (hrsg.). Soziologie und Geschichte. Band 2: Gesammelte Abhandlungen zur Soziologie, Politik und Theorie der Geschichte. 2. Aufl. Göttingen: Vandenhoeck \& Ruprecht, 1964, S. 251. Cf. dazu KOCKA, J. Historische Komparatistik in Deutschland. In: HAUPT - KOCKA, op. cit., S. $52-55$.

23 Antrittsrede von Otto Hintze in der „Königlich Preußischen Akademie der Wissenschaften“ am 2. Juli 1914. In: Sitzungsberichte, Jahrgang 1914. 2. Halbband. Berlin, 1914, S. 746; hier zitiert nach VIERHAUS, R. Otto Hintze und das Problem der vergleichenden europäischen Verfassungsgeschichte. In: BÜSCH, O. ERBE, M. (hrsg.). Otto Hintze und die moderne Geschichtswissenschaft. Berlin: Colloquium Verlag, 1983, S. 95-110 (96).

24 Hier zitiert nach BLOCH, M. Mélanges historiques. Tome I. Paris: École Pratique des Hautes Études, 1963, S. 16-40, Bibliothèque Générale de L'École Pratique des Hautes Ètudes, VIe Section; ebenso BLOCH, M. Un problème d'histoire comparée: La ministérialité en France et en Allemagne. Revue Historique de Droit Français et Ètranger, 1928, 4. série, 7, S. 46-91.

25 BLOCH, Pour une histoire comparée. In: BLOCH, M. Mélanges historiques, S. 17.

26 Cf. KAELBLE, H. Der historische Vergleich. Eine Einführung zum 19. und 20. Jahrhundert. Frankfurt am Main: Campus Verlag, 1999, S. 12 ss.

27 BLOCH, Pour une histoire comparée, S. 16.

28 Ibidem.

29 Cf. z.B. GRABOVSZKI, E. Vergleichende Literaturwissenschaft für Einsteiger. Wien (u.a.): UTB GmbH, 2011, S. 101: Kritik an der Methode der Vergleichung. 
könne, was in seiner Konstruktion bereits angelegt sei. ${ }^{30}$ Es gibt auch Stimmen, die der vergleichenden Methode „,nur wenig genuine Erklärungskraft“ zugestehen ${ }^{31}$ oder diese als die „Sache einer Minderheit“ bezeichnen. ${ }^{32}$ Auch die moderne Rechtsvergleichung schwankt zwischen Definitionsnot und Methodenzweifel. Der berühmte Rechtsvergleicher Max Rheinstein fragte 1952: “... but, then, what is it?”33 Auch Alan Watson beginnt 1974 seinen bekannten „Approach to Comparative Law“ mit der Frage: "What is Comparative Law. The question is often put: the range of replies is startling. An extreme but not uncommon opinion is that it does not exist. ... More than one comparative lawyer ... has observed that 'Comparative Law' is a strange phrase." 34

In diesem Sinne betonte Marc Ancel 1969: „... la difficulté, sinon même l'impossibilité de donner une définition unique et indiscutable de cette expression aussi attachante qu'ambiguë ... Recherche et méthode sont deux expressions aujourd'hui courantes. "35

Der Selbstzweifel der Juristen betrifft vor allem die Frage, ob die Rechtsvergleichung eine Methode ${ }^{36}$ oder eine eigenständige Disziplin ${ }^{37}$ ist. Mathias Reimann stellte die Frage „Vergleich: Fluch oder Segen?“38 Ihre von Gutteridge so bezeichnete „elastic method“39 erlaubte es, fremde Rechte unter verschiedenen Gesichtspunkten zu unterschiedlichen Zwecken zu untersuchen. Namentlich das Verhältnis zwischen Rechtsvergleichung und Rechtsgeschichte wird thematisiert und auf seine wechselseitig produktiven Erkenntnismöglichkeiten hinterfragt. ${ }^{40}$ Die Frage nach dem Nutzen der Rechtsvergleichung für die Rechtsgeschichte wird jedenfalls nicht einheitlich beantwortet. ${ }^{41}$ So hat z.B. Albrecht Cordes den Ertrag der Rechtsvergleichung für die mediävistische Rechtsgeschichte eher

30 Cf. ESPAGNE, M. Les transfers culturels franco-allemands. Paris: Presses universitaires de France, 1999, S. 35-49; Dazu WERNER, M. - ZIMMERMANN, B. Vergleich, Transfer, Verflechtung. Der Ansatz der Histoire croisée und die Herausforderung des Transnationalen. Geschichte und Gesellschaft, 2002, 28, S. 610 .

31 WELSKOPP, Th. Stolpersteine auf dem Königsweg. Methodenkritische Anmerkungen zum internationalen Vergleich in der Gesellschaftsgeschichte. AfS, 1995, 35, S. 363; PAULMANN, J. Internationaler Vergleich und interkultureller Transfer. Zwei Forschungsansätze zur europäischen Geschichte des 18. bis 20. Jahrhunderts. $H Z, 1998,267$, S. 650 ss., 682 ss.

32 HAUPT - KOCKA, op. cit., S. 23.

33 RHEINSTEIN, M. Teaching Tools in Comparative Law. American Journal of Comparative Law, 1952, 1, S. 95.

34 WATSON, A. Legal Transplants. An Approach to Comparative Law. 2. ed. Athens - London: University of Georgia Press, 1993, S. 1.

35 ANCEL, M. Réflexions sur la recherche et sur la méthode comparatives. In: V. CAEMMERER, E. et al. (hrsg.). Ius Privatum Gentium I. Festschrift für Max Rheinstein zum 70 Geburtstag am 5. 7. 1969. Tübingen: Mohr Siebeck, 1969, S. 211.

36 So z.B. ZWEIGERT, op. cit., S. 334: „Die Rechtsvergleichung ist eine Methode der Rechtswissenschaft, welche die Mythen des nationalen Rechtsstolzes abbaut", indem sie Lösungen in Rechtsordnungen anderer Länder nach ihrer Brauchbarkeit untersucht; ebenso ZWEIGERT, K. - KÖTZ, H. Einführung in die Rechtsvergleichung auf dem Gebiete des Privatrechts. Band I: Grundlagen. 2. ed. Tübingen: J. C. B. Mohr, 1984, S. 15. SCHNITZER, A. F. Vergleichende Rechtslehre. Basel: Verlag für Recht und Gesellschaft, 1945, S. 17; GUTTERIDGE, H. C. Comparative Law. 2. ed. Cambridge: Cambridge University Press, 1949, S. 5 (n. 2). REIMANN, M. Rechtsvergleichung und Rechtsgeschichte im Dialog. Zeitschrift für Europäisches Privatrecht (ZEuP), 1999, 7, S. 496-512 (S. 500).

39 GUTTERIDGE, op. cit., S. 7.

40 Cf. GENZMER, E. Zum Verhältnis von Rechtsgeschichte und Rechtsvergleichung. Archiv für Rechts- und Sozialphilosophie, 1954/55, 41, S. 326-347; REIMANN, op. cit.

41 Cf. die zusammenfassende Bewertung bei REIMANN, op. cit., S. 504-509. 
gering eingeschätzt und den Nutzen mehr bei der vergleichend arbeitenden Geschichtswissenschaft und Rechtsethnologie gesehen. ${ }^{42}$

Trotz all dieser uneinheitlichen und unsicheren Bewertungen wird in den historischen Wissenschaften und im geltenden Recht die Vergleichung überall diskutiert und praktiziert. Sie gilt als ein heuristisches Prinzip und Instrument für historisches Verstehen und Erklären. ${ }^{43}$ Gerade die Unsicherheit des Verfahrens und die Suche nach einem gesicherten Erkenntniswert hat heute eine Vielzahl von komparatistischen Varianten mit unterschiedlichen Bezeichnungen hervorgebracht, wie den „transkulturellen Vergleich“" ${ }^{44}$ den ,internationalen Vergleich“ und ,interkulturellen Transfer“ 45 die „connected, shared and entangled histories“, 46 die ,,histoire croisée“ ${ }^{47}$ „,histoire totale“, die Verflechtung, ,transnationale“ Betrachtung, „Translokalität“",48 „Rezeptionskultur“, 49 ,Transplantation“50 und den „Rechtstransfer“, den Hans Schlosser noch 2012 mit dem traditionellen Begriff der „Rezeption“ gleichgesetzt hat. ${ }^{51}$ Die drei letzteren Bezeichnungen sind vor allem im Bereich der Rechtsgeschichte gebräuchlich, wobei parallel zur heutigen „Globalisierung“ die Rechtsgeschichte sich zur „Globalgeschichte“ über Europa hinaus ${ }^{52}$ ausweitet oder auszugreifen versucht. Als Stichworte dienen besonders der individualisierende „Kontrastvergleich" und der universale historische Vergleich. ${ }^{53}$ Dabei geht es auch um die Wechselwirkungen zwischen Globalisierung und Fragmentierung, zwischen kleinen Räumen und Weltregion sowie um globale Systemzusammenhänge.

\section{Die Naturwissenschaften als Leitbild empirischer Vergleichung für die Geisteswissenschaften}

Diese mehr verwirrende als klärende Diskussion über Vergleichsgegenstände, Vergleichsziele und -techniken sowie deren Bezeichnungen und begriffliche Festlegungen muss man vor Augen haben, wenn man die Praxis der historischen Vergleichung in den Wissenschaften betrachtet. Die Vielzahl der Monographien, Aufsätze und Diskussionsebenen spiegelt

42 CORDES, A. Was erwartet die (mittelalterliche) Rechtsgeschichte von der Rechtsvergleichung und anderen vergleichend arbeitenden Disziplinen? ZEuP, 1999, 7, S. 544-552.

43 KAELBLE, op. cit., S. 12 ss.

44 HÖFERT, A. Europa und der Nahe Osten: Der transkulturelle Vergleich in der Vormoderne und die Meistererzählungen über den Islam. HZ, 2008, 287, S. 561 ss.; OSTERHAMMEL, J. Transkulturell vergleichende Geschichtswissenschaft. In: HAUPT - KOCKA, op. cit., S. 271-313.

45 PAULMANN, J. Neue historische Literatur. Internationaler Vergleich und interkultureller Transfer. Zwei Forschungsansätze zur europäischen Geschichte des 18. bis 20. Jahrhunderts. HZ, 1998, 267, S. 649-685; KAELBLE, H. - SCHRIEWER, J. (eds.). Vergleich und Transfer. Komparatistik in den Sozial-, Geschichtsund Kulturwissenschaften. Frankfurt am Main - New York: Campus Verlag, 2003.

46 Cf. die Literatur-Übersicht bei HADLER, F. - MIDDELL, M. Auf dem Weg zu einer transnationalen Geschichte. Comparativ, 2010, 20, S. 8 ss.

47 WERNER - ZIMMERMANN, op. cit., S. 607 ss.

48 Beispiele und Bewertungen bei MIDDELL, op. cit., S. 7-41; ESPAGNE, M. Kulturtransfer und Fachgeschichte der Geisteswissenschaften. In: MIDDELL, op. cit., S. 42-61.

49 MIDDELL, op. cit., S. 20.

50 WATSON, op. cit.

51 SCHLOSSER, H. Neuere Europäische Rechtsgeschichte. München: C. H. Beck, 2012, S. 8.

52 SIEDER, R. - LANGTHALER, E. (eds.). Globalgeschichte 1800-2010. Wien - Köln - Weimar: Böhlau Verlag, 2010, darin S. 9-36: Was heißt Globalgeschichte? Zuletzt zu diesem Thema: DUVE, T. What is global legal history? Comparative Legal History, 2020.

53 So z.B. KAELBLE, op. cit., S. 32 ss. 
die Vitalität des Themas wider, lässt aber keinen bestimmten Ertrag und keine gefestigte Methode erkennen. Die Debatte ist in den Geschichtswissenschaften teilweise von Skepsis und kritischen Haltungen geprägt. ${ }^{54}$ Der Vergleich ist jedoch andererseits anerkannt als ein Prüfungsinstrument, das Beobachtungen und Wahrnehmungen in der kulturellen Welt für das Verstehen von Organisationsstrukturen nutzt, wenn man auf gesellschaftliche Entwicklungen, Recht, Staat und Rechtskulturen blickt. Mein Blick richtet sich auf historische Beispiele und Strategien solcher Vergleichungstraditionen besonders des 17. und 18. Jahrhunderts. Dabei geht es nicht um eine isolierte Wissenschaftsgeschichte der Jurisprudenz oder des ,ius publicum“, sondern auch um den Kontext der anderen Wissenschaftsdisziplinen, die die vergleichende Beobachtung viel früher als die Rechtswissenschaft praktiziert haben. Vergleichung lässt sich im Zeitalter der Aufklärung - oder der Lumière und des Enlightenment - als Ausdruck eines pragmatisch-utilitaristischen Rationalismus bezeichnen. Die Natur wurde zum Gegenstand vieler Untersuchungen über den Menschen und die ihn umgebenden Welt. Dahinter stand das Ziel, Erkenntnisse über Gesetzmäßigkeiten zu gewinnen, um die soziale Ordnung in Staat und Gesellschaft nach rationalen Kriterien neu organisieren zu können. Alles, was in der physikalischen Welt erfahrbar war, sollte erkannt und ergründet werden. ${ }^{55}$ Wissenschaftlich-empirische Beobachtungen dienten dazu, allgemeine Naturgesetze aufzudecken, wie sie durch Galilei, Newton und Harvey formuliert wurden. Darin sahen die Geisteswissenschaften das Ideal größtmöglicher Gewissheit verwirklicht, die auch die Rechtswissenschaft auf der Suche nach dem ,ius certum “ und der „certa lex“ erreichen wollte. ${ }^{56}$ Die Mathematik wurde von Christian Wolff für die Rechtsphilosophie als rationale Erkenntnismethode benutzt. ${ }^{57}$ Diese war auf Messungen angewiesen, die erst die Vergleichung wiederkehrender naturwissenschaftlicher Vorgänge ermöglichte. ${ }^{58}$ Sie wurde zu einem allgemeinen Erkenntnisprinzip - auch für die geistige Welt. 1753 schreibt der Genfer Naturforscher und Philosoph Charles Bonnet an Montesquieu, nachdem dessen „De 1'Ésprit des Lois“ erschienen war: „Newton hat die Gesetze der materialen Welt aufgedeckt, und Sie haben die Gesetze der Geisteswelt aufgedeckt. “59 1769 notiert der Göttinger Physiker, Philosoph, Aufklärer und kritische Beobachter Georg Christoph Lichtenberg in seinen „Sudelbüchern“: „Eine genaue Betrachtung der äußeren Dinge führt leicht auf den betrachtenden Punkt, uns selbst, zurück und umgekehrt... Sei

54 Z.B. WERNER - ZIMMERMANN, op. cit., S. 610; HADLER - MIDDELL, op. cit., S. 9.

55 Cf. SCHNABEL, F. Deutsche Geschichte im 19. Jahrhundert. 3. Band: Erfahrungswissenschaften und Technik. Freiburg: Herder, 1934, S. 174 ss.

56 Cf. MOHNHAUPT, H. „Lex certa“ und,,ius certum“: The Search for Legal Certainty and Security. In: DASTON, L. - STOLLEIS, M. (eds.). Natural Law and Laws of Nature in Early Modern Europe. Jurisprudence, Theology, Moral and Natural Philosophy. Aldershot (et al.): Routledge, 2008, S. 73-88.

57 WOLFF, Ch. Die Anfangs-Gründe aller Mathematischen Wissenschaften Erster Theil, Welcher einen Unterricht von der Mathematischen Lehr-Art ... in sich enthält, neue ... Auflage. Franckfurt - Leipzig, 1750, S. III, VIII.

58 COING, H. Europäisches Privatrecht I: Älteres Gemeines Recht (1500-1800). München: C. H. Beck, 1985, S. 46, 69; WIEACKER, F. Privatrechtsgeschichte der Neuzeit. 2. Aufl. Göttingen: Vandenhoeck u. Ruprecht, 1967, S. 254 ss.

59 Hier deutsch zitiert nach LARRÈRE, C. In Search of the Newton of the Moral World... In: DASTON STOLLEIS, op. cit., S. 249 (dort n. 6). 
aufmerksam, empfinde nichts umsonst, messe und vergleiche: dies ist das ganze Gesetz der Philosophie." 60

Es war ein Ziel der Aufklärungsphilosophie, mit Hilfe von ermittelten Erfahrungssätzen auf gesellschaftliche Prozesse Einfluss zu nehmen und - wie von Rochow 1777 erklärte durch ,moralische Ursachen, Regeln und Gesetze ...(das) Verhalten zu bestimmen und zu einer gewissen Absicht zu leiten."61

In diesem Sinne hatte Paul Henri Thiry d'Holbach 1770 in seinem „Systême de la nature, ou, des Loix du monde physique et du monde moral“" programmatisch für Erfahrung und Beobachtung in den Wissenschaften plädiert: „Der Mensch wollte Metaphysiker seyn, ehe er Physiker war ..., (er) verachtete die Erfahrung, um sich an Lehrgebäude und Muthmaßungen zu hängen. Er wagte es nicht seine Vernunft anzubauen ... Mit einem Worte, der Mensch verachtete die Beobachtung der Natur, um Phantomen nachzulaufen." 62 „...Physik und Erfahrung ... sind es, die er bey seiner Religion, seiner Moral, seiner Gesetzgebung, seinen Regierungsformen, seinen Wissenschaften ... zu Rathe ziehen muß. Die Natur wirkt nach einfachen, gleichförmigen, unveränderlichen Gesetzen; und die Erfahrung setzt uns in den Stand, diese zu erkennen ... und ihre Geheimnisse (zu) entdecken."63 Und er fährt fort, indem er den Menschen selbst als Gegenstand der Vergleichung sieht und bezeichnet: „,(Die) Eigentümlichkeit des Menschen, ... zu empfinden, zu denken, zu handeln ..., welche ihn von allen anderen Wesen, mit welchen er sich vergleicht, unterscheidet. Dieser Vergleichung zu Folge ... (ist) der Mensch ein System, ... eine besondere Classe...".64

\section{Die Vergleichsziele bestehen in der Aufdeckung von Gesetzmäßigkeiten in der belebten und unbelebten Welt}

In den Naturwissenschaften wurde der Vergleich in einer systematischen Form viel früher praktiziert als in den Geisteswissenschaften. 1675 legte der Engländer Nehemiah Grew der „Royal Society“ eine wissenschaftliche Untersuchung vor, die den Titel trug: „The Comparative Anatomy of Trunks, Together with an Account of their Vegetation grounded thereupon“. 65 Der anatomische Vergleich betraf Pflanzen, Tiere und Menschen. In seiner Widmung an den englischen König betonte Grew das Erkenntnisziel des „Universal search of Truth“ mit den Worten: „In sum, Your Majesty will find, that we are come ashore into a new World, whereof we see no end."66

60 LiCHTENBERG, G. Chr. Schriften und Briefe. 1. Band: Sudelbücher. München: Carl Hanser Verlag GmbH \& Co. KG, 1968, S. 38.

61 VON ROCHOW, F. E. Entwurf der allgemeinen Grundsätze der Gesetzgebung. Frankfurt - Leipzig, 1777 , S. 8 .

62 Hier zitiert nach der deutschen Übersetzung: D'HOLBACH, P. H. T. System der Natur, oder von den Gesetzen der physischen und moralischen Welt. 1. Band. Aus dem Französischen des Herrn Mirabeaud, Frankfurt - Leipzig, 1783, Vorerinnerung S. XII.

63 D'HOLBACH, op. cit., S. 5.

64 D'HOLBACH, op. cit., S. 11 ss.

65 GREW, N. The Comparative Anatomy of Trunks, together with an account of their vegetation grounded thereupon; in two parts: the former read before the Royal Society, Feb. 25. 1674/1675; the latter, June 17. 1675. The whole explicated by several Figures in Nineteen Copper-Plates; presented to the Royal Society in the years 1673 and 1674. London, 1675.

66 GREW, op. cit., The Epistle Dedicatory. 
An erster Stelle der Untersuchung steht die ,particular Description“ der durch das Mikroskop beobachteten Phänomene, gefolgt sodann von der eigentlichen Vergleichung: „... but also by a comparative prospect, in what respects they are specifically distinguished one from another, in the several species of Branches. “67

Später haben z.B. in Göttingen Johann Friedrich Blumenbach in seinem Specimen physiologiae comparatae von $1787^{68}$ und Georges de Cuviers in seinen „Leçons d'anatomie comparée“ von $1800^{69}$ in diesem Sinne gearbeitet. Man kann im 18. Jahrhundert von einer Strategie des Vergleichens als Erkenntnismittel sprechen. Als Beispiele sind zu nennen der Religionsvergleich bei Joseph White (1786) ${ }^{70}$ und Priestley, ${ }^{71}$ der Kulturvergleich bei Giambattista Vico, ${ }^{72}$ Wilhelm Humboldts neue Wissenschaft einer vergleichenden Anthropologie oder der Sprachenvergleich bei Court de Gébelin (1775) sowie Thurot (1797) $)^{73}$ und Adelung (1782). ${ }^{74}$ Wie Adelung behandelt auch Antoine Court de Gébelin den Sprachenvergleich als Kulturvergleich: „Monde primitif analysé et comparé avec le monde moderne, considéré dans l'histoire naturelle de la parole ou origine du langage ...".75 Ausdrücklich erklärt er den diachronen Vergleich als unverzichtbares Erkenntnismittel: „,... que sans ces comparaisons, tout travail sur les Langues est nécessairement defectueux."76

Die Strategie des Vergleichens dominierte jedoch in den Naturwissenschaften. Mit der Corpus-Metapher wurden der Staat und seine Funktionen erklärt: Corpus humanum est pariter quaedam quasi Respublica. ${ }^{77}$ Dementsprechend werden auch die Aufgaben der ,jurisprudentia“ und der ,medicina“ verglichen, denn wie der Arzt heilt auch der Jurist die „Respublica“, indem er durch Gesetze dem Staatskörper zur ,felicitas“ verhilft und

67 GREW, op. cit., S. 2.

68 BLUMENBACH, J. F. Specimen physiologiae comparatae inter animantia calidi et frigidi sanguinis. Göttingen, 1787.

69 DE CUVIER, G. Leçons d' anatomie comparée. Tom. I-V. Paris, 1800-1805.

70 WHITE, J. Vergleichung der christlichen Religion mit der mohamedanischen. Aus dem Englischen übersetzt von Johann Gottfried Burkhard. Halle, 1786.

71 PRIESTLEY, J. A comparison of the institutions of Moses with those of the Hindoos and other ancient nations with remarks on Mr. Dupuis's Origin of all religions, the laws and institutions of Moses methodized, and an address to the Jews on the present state of the world and the prophecies relating to it. Northumberland: Printed for the author by A. Kennedy, 1799; deutsche Übersetzung: Priestley's Vergleichung der Gesetze des Moses mit denen der Hinduer und anderer Nationen. Verdeutscht und mit einem erläuternden Anhange begleitet von J. W. H. Ziegenbein. Braunschweig, 1801.

72 VICO, G. Principi di una scienza nuova intorno alla natura delle nazioni per la quale si ritruovano i principi di altro sistema del diritto naturale delle genti. Napoli, 1725.

73 Cf. die Übersicht bei MOHNHAUPT, H. Historische Vergleichung im Bereich von Staat und Recht vom späten 18. bis zur Mitte des 19. Jahrhunderts. Beobachtungen zur deutschen Bezugnahme auf Italien. In: MAZZACANE - SCHULZE, op. cit., S. 31-62 (S. 43-45); Idem. Vergleichende Beobachtung von Staat, Gesellschaft und Recht im 18. Jahrhundert als Vorform der modernen Rechtsvergleichung. Comparative Law (Nihon University), 1997, Vol. 14, S. 1-24 (S. 12-17).

74 ADELUNG, J. Chr. Beweis der fortschreitenden Cultur des menschlichen Geistes aus der Vergleichung der älteren Sprachen mit den neuern. Magazin für die Deutsche Sprache, 1782, S. 3-28.

75 So der Titel, Paris, 1775.

76 COURT DE GÉBELIN, op. cit., Discours Préliminaire, S. XVIII.

77 GOELICKE, A. O. Medicina forensis demonstrativa methodo tradita cui praemissa est introductio in historiam literariam scriptorum qui medicinam forensem commentariis suis illustrarunt. Francofurti ad Viadrum, 1723, Oratio praeliminaris, s. p. (S. 10). 
dadurch kranke Glieder entfernt. Et Medicinae scopus nullus quoque alius est. ${ }^{78}$ Die vergleichend arbeitende Medizin wurde offensichtlich auch von der Jurisprudenz aufmerksam verfolgt, denn noch 1810 konnte der Jurist und Philosoph Paul Johann Anselm von Feuerbach die kritische Frage an seine juristische Disziplin stellen: „Warum hat der Anatom seine vergleichende Anatomie? Und warum hat der Rechtsgelehrte noch keine vergleichende Jurisprudenz?"79

„Vergleichung und Combination“, Aufdeckung der „Gegensätze“, „Betrachtung der Ähnlichkeiten und Verschiedenheiten“, das „Allgemeine aus dem Besonderen herauszufinden“ bildeten für ihn die wichtigsten methodischen Verfahren für ,alle Entdeckungen in jeder Erfahrungswissenschaft“. Er bezieht sich auch auf den Sprachenvergleich und fährt hier fort: „So wie aus der Vergleichung der Sprachen die Philosophie der Sprache, die eigentliche Sprachwissenschaft hervorgeht; so auch aus der Vergleichung der Gesetze und Rechtsgewohnheiten der verwandesten, wie der fremdartigsten Nationen aller Zeiten und Länder, die Universal-Jurisprudenz .... 80

Das Vergleichsziel besteht für Feuerbach darin, das „Allgemeine“ aus Unterschieden und Gegensätzlichkeiten heraus zu finden und ,in ein großes sinnvolles Ganze zusammen zu fassen“, wofür ihm Montesquieu ausdrücklich als Vorbild dient. Und wiederum mit Bezug auf die Medizin betont er: „... erst die Vergleichung der Organe aller lebenden Körper führt die Zergliederungskunst zur Wissenschaft. “81

Autoren aus der Sprachwissenschaft, Medizin oder anderen Disziplinen nennt er namentlich nicht. Als wissenschaftliches Verfahren ist jedoch die Vergleichungsmethode offensichtlich ein allgemein praktiziertes Erkenntnismittel. Feuerbach hätte sich sogar auf Goethe berufen können, der 1790 in seinem ,Versuch einer Allgemeinen Vergleichungslehre" 82 und 1795 in seiner „Allgemeinen Einleitung in die vergleichende Anatomie“"83 erklärt hatte: „Naturgeschichte beruht überhaupt auf Vergleichung.“ Sowohl bei Feuerbach als auch bei Goethe sind die entscheidenden Stichworte: Erfahrung, Vergleichung, Kombination, Entdeckung der „Organisation“ eines jeden lebenden Wesens, das Allgemeine und Besondere, die „Betrachtung von innen nach außen und von außen nach innen“ und „Fortschritt der Wissenschaft“ unter dem Gesichtspunkt des „Nutzens für die Menschen“. 84

78 GOELICKE, op. cit., Oratio praeliminaris, s. p. (S. 11).

79 VON FEUERBACH, J. P. A. Vorrede zu Carl August Dominik Unterholzner. Juristische Abhandlungen. München: E. A. Fleischmann, 1810; wieder abgedruckt unter dem Titel „Blick auf die teutsche Rechtswissenschaft“. In: Anselm von Feuerbach, Kleine Schriften vermischten Inhalts. Nürnberg, 1833, S. 163 ss. (hier nach dieser Ausgabe zitiert). Dazu MOHNHAUPT, H. Universalgeschichte, Universal-Jurisprudenz und rechtsvergleichende Methode im Werk P. J. A. Feuerbachs. In: Idem (ed.). Rechtsgeschichte in den beiden deutschen Staaten (1988-1990). Beispiele, Parallelen, Positionen (Ius Commune, Sonderhefte 53). Frankfurt am Main: Klostermann, 1991, S. 97-128.

80 VON FEUERBACH, Blick auf die teutsche Rechtswissenschaft.

81 VON FEUERBACH, P. J. A. Idee und Nothwendigkeit einer Universaljurisprudenz. Naturrecht, Rechtsphilosophie, allgemeine Rechtswissenschaft. In: Anselm Ritter von Feuerbach's Biographischer Nachlaß. Veröffentlicht von seinem Sohne Ludwig Feuerbach. 2. Band. 2. Aufl. Leipzig: J. J. Weber, 1853, S. 381.

82 GOETHE, J. W. Gedenkausgabe der Werke, Briefe und Gespräche (28. August 1949). Band 17. In: BEUTLER, E. (hrsg.). Zürich - Stuttgart, Artemis, 1964, S. 226 ss.

83 Erster Entwurf einer Allgemeinen Einleitung in die vergleichende Anatomie, ausgehend von der Osteologie. In: GOETHE, Gedenkausgabe der Werke, Briefe und Gespräche (28. August 1949), S. 28-36.

84 Den Abstand zur modernen historischen Vergleichung lässt BLOCH, Pour une histoire comparée, S. 40, deutlich erkennen, denn diese ist auf wissenschaftliche Erkenntnis gerichtet, „non vers la pratique“. 
Ich habe diese naturwissenschaftlich inspirierten Stimmen und strategischen Vergleichsziele etwas ausführlicher zitiert, da sie sich im Bereich der „Politica“ und des ,ius publicum" wiederfinden und z.T. einen Gleichklang in den Wissenschaften dokumentieren. Mit den zunehmenden naturwissenschaftlichen Erfolgen im Aufdecken von Gesetzmäßigkeiten durch das Vergleichen und Messen der natürlichen physikalischen Welten ${ }^{85}$ wurde diese Methode auch immer attraktiver für die Erforschung und Analyse des gesellschaftlichen bzw. staatlichen Körpers, um hier die von Hobbes gebrauchte „Corpus“-Metapher zu verwenden. ${ }^{86}$ Zwar hatte schon Plato für die „Nomoi“ der griechischen Stadtstaaten eine vergleichende Beobachtung gefordert, ${ }^{87}$ um daraus Erkenntnisse über die optimale Rechtsordnung zu gewinnen, und Aristoteles hatte immer wieder die Frage nach der „,besten Verfassung“ gestellt, d.h. nach der für das jeweilige staatliche Gemeinwesen geeignetsten Regierungsform unter den drei Möglichkeiten Monarchie, Aristokratie und Politie sowie deren drei negativen Abweichungen. ${ }^{88}$ Die Suche nach dem Idealstaat in der Antike betraf sowohl seine Verfassung als auch die dieser Verfassung angemessenen Gesetze. ${ }^{89}$ Diese Fragen waren immer als Komparativ mit der Suche nach dem Superlativ gestellt - also auf eine Vergleichung ausgerichtet. In der Epoche der Lumière des 17. und 18. Jahrhunderts wird jedoch das Programm der Aufklärung utilitaristisch ausgerichtet, das seine Ideen und Projekte in gesellschaftliche Praxis umzusetzen versuchte. ${ }^{90}$ Das entsprach dem Anspruch des aufgeklärten Vernunftrechts, in die Entwicklung von Gesellschaften und Staaten mit den Mitteln des Rechts gestaltend einzugreifen. Aus der Vergleichung gewonnener Erfahrungswerte wurden Gestaltungsstrategien abgeleitet und aus aufgedeckten naturwissenschaftlichen Kausalitäten folgten Möglichkeiten für deren zweckgerichtete Durchsetzung in anderen Wissenschaftsbereichen. Das spiegelte auch der Systembegriff der Enzyklopädisten wider: „... faire des systèmes: mais dans les uns, on se propose de rendre raison des effets; dans les autres, de les préparer et de les faire naître. Le premier objet est celui de la physique; le second est celui de la politique. “91

Das aufklärerische Ideal von einer Einheit der Wissenschaften bestimmte diese Auffassung. In der Vorrede zu Gundlings „Discours über ... (die) Europäischen Staaten“ heißt es 173392: „Es gibt wohl keine Wissenschaft, welche nicht von der anderen etwas abborgen sollte ...".93 Analogieschluss und Vergleich bildeten Ende des 18. Jahrhunderts in den historischen Wissenschaften sowie in der Biologie und Philologie das methodische Handwerkszeug, um theoretische und praktische Kenntnisse zu erlangen. ${ }^{94}$ Das galt auch

85 Cf. BEHRISCH, L. (hrsg.). Vermessen, Zählen, Berechnen. Die politische Ordnung des Raums im 18. Jahrhundert. Frankfurt am Main - New York: Campus, 2006.

86 Cf. auch oben, GOELICKE, op. cit.

87 PLATON. Gesetze. Buch XII, 951a-952b.

88 ARISTOTELES. Politik, 2. Buch, 1261a; 4. Buch 1288 b - 1289 a.

89 Cf. auch GENZMER, op. cit., S. 327 ss.

90 Cf. z.B. MEINEL, Chr. Reine und angewandte Chemie. Die Entstehung einer neuen Wissenschaftskonzeption in der Chemie. Berichte zur Wissenschaftsgeschichte, 1985, 8, S. 7-24.

91 Encyclopédie ou Dictionnaire Raisonné des Sciences. XIII. Genève, 1779, S. 299.

$92 \mathrm{Zu}$ Gundling und die Hallesche Schule, cf. STOLLEIS, M. Geschichte des öffentlichen Rechts I. München: C. H. Beck, 1988, S. 302-308.

93 FRANKENSTEIN, J. A. Vorrede. In: GUNDLING, N. H. Ausführlicher Discours über den jetzigen Zustand Der Europäischen Staaten. Mit ...einer Vorrede ... Franckfurt - Leipzig, 1733, s.p. (S. XIV).

94 REILL, P. H. Die Geschichtswissenschaft um die Mitte des 18. Jahrhunderts. In: VIERHAUS, R. (hrsg.). Wissenschaften im Zeitalter der Aufklärung. Göttingen: Vandenhoeck \& Ruprecht, 1985, S. 189-191. 
und besonders für die sogenannte „Staaten-Lehre“, die mit der ,allgemeinen Politic, der Staats-Rechts-Lehre, der Historie und Geographie ..." verbunden wurde. Hier liegt der Schwerpunkt der folgenden Ausführungen.

\section{Die Vergleichungen auf den verschiedenen Feldern des Rechts als Reflex eines verwirrenden Rechtsquellenpluralismus und der wachsenden Vielfalt der Staatenwelt}

Im Bereich des Rechts betrafen die Vergleichungen das ,ius privatum“, die „Politica“, das „,ius publicum“ und die ,prudentia legislatoria“ mit unterschiedlicher Akzentuierung. ${ }^{95}$ Nicht alles ist Vergleichung, was als Vergleich bezeichnet wurde und viele Lehrbücher und Dissertationen bezweckten Vergleichung, ohne so bezeichnet zu sein. „Compartio“, „Vergleich“, „collatio“, „conferendum est", „,parallele or conference“, „,ifferentia“, „aequitas“, ,similitudo“, „analogia“ und „,comparaison“ sind die häufig gebrauchten Begriffe, die auf vergleichende Beobachtung zielen. Ohne eine einheitliche Methode zu entwickeln, besteht der gemeinsame Zweck darin - um noch einmal mit Feuerbach zu sprechen - durch die ,,vergleichende Kenntniß des Verschiedenen“ zur „Erkenntniß des Allgemeinen und Nothwendigen“" zu gelangen. ${ }^{96}$

Für das Privatrecht - aber nicht nur für diese Rechtsdisziplin - verfolgt die moderne Rechtsvergleichung hauptsächlich das Ziel, unter den verglichenen dogmatischen Rechtsinstituten, staatlichen Rechtsordnungen und Rechtskulturen für ein Rechtsproblem die beste Lösung zu finden, d.h. aus dem Bestand verglichener, vorfindbarer Lösungen die am meisten geeignete und angemessene auszuwählen und anzuwenden. ${ }^{97}$ Für die Arbeit des Rechtsvergleichers können heute drei Aufgaben benannt werden: die instrumentale, die rechtskulturelle und die der „Koordinierung von nationalen und supranationalen Rechtsordnungen". ${ }^{98}$ Elemente der beiden ersten Aufgabenstellungen lassen sich auch in der historischen Vergleichspraxis erkennen. In der Privatrechtswissenschaft des 17. und 18. Jahrhunderts ist die vergleichende Beobachtung vor allem auf die „confusiones“ des römischen Rechts gerichtet, an denen sich die Kritik an den verwirrenden Rechtszuständen entzündete. Diese betraf grundsätzlich alle Länder des europäischen ,ius commune“ mit dem Ziel, „par la comparaison“ „porter la lumière dans le labyrinthe de nos Coutumes, et à dépouiller 1' étude de notre Droit. "99 Es ging darum, die Unterschiede und Gemeinsamkeiten

95 Dazu MOHNHAUPT, H. Vergleichung in Zeiten des Naturrechts der Aufklärung als Erkenntnismethode. In: ARMGARDT, M. - REPGEN, T. (hrsg.). Naturrecht in Antike und früher Neuzeit. Symposion aus Anlass des 75. Geburtstages von Klaus Luig. Tübingen: Mohr Siebeck, 2014, S. 92-114, worauf hier teilweise Bezug genommen wurde.

96 VON FEUERBACH, op. cit., S. 382.

97 ZWEIGERT, op. cit., S. 341; COING, H. Die juristischen Auslegungsmethoden und die Lehren der allgemeinen Hermeneutik. In: Arbeitsgemeinschaft für Forschung des Landes Nordrhein-Westfalen, Geisteswissenschaften. Heft 14. Köln: VS Verlag für Sozialwissenschaften, 1959, S. 21; DROBNIG, U. Methodenfragen der Rechtsvergleichung im Lichte der „International Encyclopedia of Comparative Law“. In: VON CAEMMERER, E. et al. (hrsg.). I us Privatum Gentium. Festschrift für Max Rheinstein. Band I. Tübingen: Mohr (Siebeck), 1969, S. 225; UTERMARK, T. Rechtsgeschichte und Rechtsvergleichung bei Ernst Rabel. Frankfurt am Main (et al.): Peter Lang, 2005, S. 121 ss.

98 So BUXBAUM, R. M. Die Rechtsvergleichung zwischen nationalem Staat und internationaler Wirtschaft. Rabels Zeitschrift für ausländisches und internationales Privatrecht, 1996, 60, S. 205-211.

99 So z.B. der programmatische Titel von GIN, M. Analyse Raisonnée du Droit François, par la comparaison des dispositions des Loix Romaines, et de celles de la Coutume de Paris ... Paris, 1782, mit préface. 
zwischen dem römischen ,ius commune“ auf der einen Seite und den zahlreichen partikularen Privilegien, ,iura singularia“, Gewohnheitsrechten sowie Territorial-, Regional- und Lokalrechten auf der anderen Seite festzustellen, um diese harmonisieren und möglichst vereinheitlichen zu können. Diese Rechtskritik, Bestandsaufnahmen und Reformbemühungen sind Vorarbeiten für generalisierende Gesetzgebungen, für die das Instrument der Vergleichung eingesetzt werden konnte. Das geschah durch die in Gesamteuropa verbreitete „Differentienliteratur“. Vier Tendenzen werden bei der Analyse und Stoffordnung der unterschiedlichen Rechtsquellenebenen erkennbar:

1. Die Harmonisierung divergierender Regelungen innerhalb des ,ius commune“.

2. Eine Klärung des Kollisionsverhältnisses zwischen „ius commune“ und heimischem ,ius patriae“.

3. Die Vereinheitlichung und Harmonisierung innerhalb bestehenden Partikularrechts. Ein Motto lautete: Von der „coutume“ zur „,coutume generale“ oder von der „,consuetudo particularis“" zur „consuetudo generalis ${ }^{\text {“6 }}$.

4. Auf der Grundlage der bestehenden partikularen Rechte und des gemeinen Rechts die Schaffung eines neuen staatlichen Einheitsrechts in der Gestalt der Kodifikationen der Aufklärungsepoche. Ein Stichwort lautet: Durch „,Vergleichung“ zur „Gleichheit““. 100 Die Auflösung der Konflikt- und Kollisionsverhältnisse konnte methodisch nur mit Hilfe der Vergleichung bewältigt werden. Im „Lexicon philosophicum“ des Goclenius von 1613 ist dieses Arbeitsprinzip allgemeingültig so formuliert: „Accipitur generaliter (comparatio) pro aliquorum inter se collatione." 101 Die Differentienliteratur ist ein Anwendungsfall dieser „comparatio“. Die Wurzeln dieses Verfahrens gehen bis in die Antike und das Spätmittelalter zurück: „Tum ex collatione facta apparebit tibi lux quaedam mirabilis. "102

Die vergleichende Feststellung der Unterschiede umfasste fast alle Rechtsbereiche. ${ }^{103}$ Die ,differentiae iuris communis et statutorum “ betrafen Vergleichungen einzelner Probleme des römischen Erbrechts und Vertragsrechts gegenüber den partikularen Rechten. Unterschiede zwischen ,ius civile“ und ,ius canonicum" wurden untersucht. Das ,ius patriae“ als ein „ius particulare“ gegenüber dem ,ius commune“ wurde neu vermessen. Das galt auch für die Ermittlung der ,differentiae iuris publici et civilis“. ${ }^{104}$ Man kann diese privatrechtlichen Vergleichungen auch als wissenschaftliche Vorbedingungen, für die im 18. Jahrhundert viel diskutierte und verwirklichte Kodifikation ansehen, d.h. das systematische einheitliche Gesetzbuch, wie es in Preußen (1794), Frankreich (1804) und Österreich (1811) zuerst geschaffen wurde. Vereinheitlichung und Generalisierung des Rechts konnten nur auf der Grundlage, der im Vergleich erfassten, unterschiedlichen Rechtsquellen und Rechte erfolgen. Ausländische Rechte und Gesetze wurden gleichfalls in diese

100 MOHNHAUPT, H. Die Differentienliteratur als Ausdruck eines methodischen Prinzips früher Rechtsvergleichung. In: DURAND, B. - MAYALI, L. (eds.). Excerptiones iuris: Studies in Honor of André Gouron. Berkeley: Robbins Collection, School of Law, 2000, S. 439-458 (440).

101 GOCLENIUS, R. Lexicon philosophicum, quo tanquam clave philosophiae fores aperiuntur. Francofurti, 1613, S. 416.

102 OLDENDORP, J. Collatio iuris civilis et canonici, maximam adferens boni et aequi cognitionem. Coloniae, 1541, S. III (Epistola nuncupatoria).

103 Differentiarum in iure libri duo. Item definitonum ex utroque iure, centuriae VII. iuxta seriem literarum collocatae, edita per Georgium Lauterbegum. Basileae, 1551.

104 Cf. die kurze Übersicht bei MOHNHAUPT, Die Differentienliteratur als Ausdruck eines methodischen Prinzips früher Rechtsvergleichung, S. 453-458. 
vergleichenden Beobachtungen einbezogen. Die ,collationes et differentiae iuris civilis et iurium exterorum" bildeten einen besonderen Fall länderübergreifender Vergleichungen, um - wie es Bernhöft 1878 für die vergleichende Rechtswissenschaft erklärt hatte - ,die beiden Rechte ... nach Ähnlichkeit und Verschiedenheit ... gründlich zu untersuchen, und zugleich im Auslande sorgfältige Umschau zu halten, um fremde Vorzüge nachahmen, fremde Fehler vermeiden zu lernen." 105

\section{Universalismus als Ziel und Erkenntnis aus Vergleichung in der öffentlichen Sphäre}

Die Vergleichung bot ein Kriterium für Wissenschaftlichkeit, nämlich Gesetzmäßigkeiten und Regelmaß im Sinne des aufgeklärten Gleichheitsideals und Nützlichkeitsgebotes für die Verwirklichung des „,bonum publicum“, des „bien commun“ oder der „felicité“ aufzudecken. Das Allgemeine und Gleiche - durch einen Abstrahierungsprozess aus ermittelten Unterschieden gewonnen - war ein Ergebnis von Vergleichungen und repräsentierte aufklärerische ,universalitas“, die Friedrich Schiller „die Gleichförmigkeit und unveränderliche Einheit der Naturgesetze“ nannte. ${ }^{106}$ Die Bezeichnungen wie die ,monarchia universalis“, 107 die ,jurisprudentia universalis"108 und das ,ius publicum universale“109 belegen diesen Versuch einer Überwindung des Partikularen, der kosmopolitisch angelegt ist und in Ländergrenzen nur Beschränkungen des Erkenntnisprozesses sieht. Heute würde man von Globalisierung sprechen. Im 18./19. Jahrhundert zeigt sich diese Tendenz in den Wortkombinationen von „Weltgeschichte“ bei Schiller ${ }^{110}$ und Hegel, ${ }^{111}$ „Allgemeine Welthistorie“, 112 „Weltbegebenheiten, Weltverfassung, Weltveränderungen, Weltordnung“ gleichfalls bei Schiller, ${ }^{113}$ der von unserem Kontinent als dem „kleinen Weltteil Europa“ spricht. ${ }^{114}$ Solche Beispiele bieten auch die Geschichte ,in weltbürgerlicher Absicht“ bei Kant, ${ }^{115}$ das „Weltbürgerrecht“ bei Zachariae, ${ }^{116}$ die bekannten Wortschöpfungen „Weltliteratur“" und „Weltgesellschaft“ bei Goethe, der „Weltgeist der Aufklärung“ bei Hegel, das

105 BERNHÖFT, F. Ueber Zweck und Mittel der vergleichenden Rechtswissenschaft. In: BERNHÖFT, F. COHN, G. (hrsg.). Zeitschrift für vergleichende Rechtswissenschaft. Band 1. Stuttgart: Ferdinand Enke, 1878, S. 1-38 (4).

106 SCHILLER, F. Was heißt und zu welchem Ende studiert man Universalgeschichte? Eine akademische Antrittsrede. In: Der Teutsche Merkur. Weimar, 1789. Repr. Jena: Verlag Dr. Bussert \& Stadeler, 1996, S. 129 ss.; cf. dazu HEUER, F. Spuren der Universalgeschichte in Schillers Jenaer Umkreis: Der Fall von Karl Ludewig Woltmann. In: STRACK, F. (hrsg.). Evolution des Geistes: Jena um 1800. Stuttgart: Klett-Cotta, 1994, S. 132-155.

107 GUNDLING, op. cit., S. 22 ss.

108 Z.B. PUFENDORF, S. Compendium iurisprudentiae universalis ... Francofurti, 1694.

109 Z.B. BÖHMER, J. H. Introductio in ius publicum universale ... Halae Magdeburgicae, 1710; dazu STOLLEIS, op. cit., S. 268 ss.

110 SCHILLER, op. cit., S. 120, 132.

111 HEGEL, G. W. Vorlesungen über die Philosophie der Geschichte. In: MOLDENHAUER, E. (hrsg.). Werke. Band 12. Frankfurt am Main: Suhrkamp, 1973, S. 105 ss.

112 Uebersetzung der Allgemeinen Welthistorie in England durch eine Gesellschaft von Gelehrten ..., durchgesehen ... Von S. J. Baumgarten. Halle, 1744.

113 SCHILLER, op. cit., S. 122, 127, 128, 131, 132.

114 Ibidem, S. 121.

115 KANT, I. Ideen zu einer allgemeinen Geschichte in weltbürgerlicher Absicht (1784).

116 ZACHARIAE, K. S. Die Wissenschaft der Gesetzgebung. Als Einleitung zu einem allgemeinen Gesetzbuche. Leipzig, 1806, S. 169. 
im Hegelschen Sinne philosophisch inspirierte „Erbrecht in weltgeschichtlicher Entwicklung“"von Eduard Gans, ${ }^{117}$ die ,Weltrechtsgeschichte“ bei Karl Theodor Pütter, ${ }^{118}$ die ,allgemeine Weltidee“ bei Albert Hermann Post (1866), ${ }^{119}$ eine ,Welt Rechts Wissenschaft“ bei Saleilles ${ }^{120}$ und schließlich Ende des 19. Jahrhunderts Ernst Zitelmanns Frage nach der „Möglichkeit eines Weltrechts““.121 Ganz im Sinne dieser bezeichneten kosmopolitisch ausgerichteten Sicht hatte 1856 Johannes Emil Kuntze einen „Fortschritt“ der „Methode“ festgestellt, „daß entschiedener als bisher der Horizont unserer Wissenschaft zu einer komparativen Jurisprudenz im umfassenden Weltsinne ausgeweitet werde."122

Diese universalistische Betrachtungsweise mit dem Versuch, Recht in seiner Totalität als weltweites Kulturphänomen vergleichend zu erfassen und zu gestalten, hat Wurzeln im Geist der Aufklärung. Besonders die Staatenwelt des 17./18. Jahrhunderts war im Zeitalter des Vernunftrechts ein Vergleichsobjekt mit einer bevorzugt europäischen Dimension, die aber auch teilweise auf außereuropäische Regionen und Länder ausgedehnt wurde. Bereits 1572 hatte Jean Bodin die „Comparatio Rerumpublicarum“ ganz im Stil der aristotelischen Staatsformenlehre vorgenommen. ${ }^{123}$ Solche Untersuchungen waren nur mit einem vergleichenden Blick auf andere Staaten möglich, um Erfahrungswerte zu gewinnen, welche Staats- bzw. Regierungsform für einen Herrschaftsbereich als die angemessenste gelten könne. Typisch für diese Prüfungsfrage ist die Untersuchung von Besold in seinem Discursus quintus, in quo rerumpublicarum formae inter sese comparantur: illarum praestantiae certamen examinatur. Cap. I: De Monarchiae. Democratiae et Aristocratiae praerogativa, quam singulae habere videntur. ${ }^{124}$

1682 hat Pufendorf in seiner „Einleitung zu der Historie der vornehmsten Reiche und Staaten“" eine umfassende vergleichende Übersicht gegeben, ${ }^{125}$ die historische und aktuell

117 GANS, E. Das Erbrecht in weltgeschichtlicher Entwicklung. Band I-IV. Berlin - Stuttgart - Tübingen: Mauersche Buchhandlung - J. B. Cotta'schen Buchhandlung, 1824-1835; cf. dazu MOHNHAUPT, H. Universalrechtsgeschichte und Vergleichung bei Eduard Gans. In: BLÄNKNER, R. et al. (hrsg.). Eduard Gans (1797-1839). Politischer Professor zwischen Restauration und Vormärz. Leipzig: Leipziger UniVlg, 2002, S. 339-366; BERTANI, C. Das Erbrecht in weltgeschichtlicher Entwicklung (1824-1835) von Eduard Gans. $R G, 2007,11$, S. 110-138.

118 PÜTTER, K. Th. Der Inbegriff der Rechtswissenschaft oder Juristische Encyclopädie und Methodologie. Berlin: De Gruyter, 1846, S. VIII, XX.

119 Cf. KIESOW, op. cit., S. 51.

120 SALEILLES, R. Le code civil et la Méthode historique, hier zitiert nach GERGEN, Th. Wie sahen französische Juristen die Rezeption ,,ihres“ Code civil mit besonderem Bezug auf Deutschland? In: KERN, B.-R. (hrsg.). Code civil. Leipzig: Leipziger Universitätsverlag, 2010, S. 78.

121 ZITELMANN, E. Die Möglichkeit eines Weltrechts. Wien: Manz, 1888.

122 KUNTZE, J. E. Der Wendepunkt der Rechtswissenschaft; ein Beitrag zur Orientierung über den gegenwärtigen Stand- und Zielpunkt derselben. Leipzig: Hinrichs, 1856, S. 10 (Hervorhebung bei Kuntze); ähnlich LENZ, G. Ueber die geschichtliche Entstehung des Rechts. Eine Kritik der historischen Schule. Greifswald - Leipzig: Koch, 1854, S. 21, dass - hier Eduard Gans folgend - „,ein allgemeines Streben nach Vergleichung erwacht sei“.

BODIN, J. Methodus ad facilem historiarum cognitionem; ab ipso recognita ... Parisiis, 1572. In: MESNARD, P. Oeuvres Philosophiques de Jean Bodin. Paris: Presses universitaires de France, 1951, S. 182 (nr. 45); zum „,comparatisme“ cf. BEAUD, O. La Puissance de l'État. Paris: Presses Universitaires de France, 1994, S. 56 ss.

124 BESOLD, Chr. Discursus politici. Argentorati, 1642, S. 182 ss.

125 PUFENDORF, S. Einleitung zu der Historie der Vornehmsten Reiche und Staaten, so itziger Zeit in Europa sich befinden. Franckfurt am Mayn, 1782. 
zuständliche Eigenschaften fast aller Länder zusammenfasste. Dieses Buch wurde in viele Landessprachen übersetzt und erfüllte offensichtlich ein Informationsbedürfnis der politischen Welt. Pufendorfs Werk wurde zu einem Modell für die europäische Literaturgattung der sogenannten „Notitia rerum publicarum“ und der „Statistik“. ${ }^{126}$ Nach einem unterschiedlich weit gefassten Kriterienkatalog werden z.B. „origo et progressus civitatis“, „,administratio summi imperii“, ,forma reipublicae“, natürliche Ressourcen, ${ }^{127}$ Stärken und Schwächen ${ }^{128}$ sowie die „Grundverfassung“129 der europäischen Staaten ermittelt und aufgelistet, um aus diesem Datenkatalog die „optima respublica“ und das jeweilige „Staats-Interesse“ ableiten und entwickeln zu können. Die rechtlichen Bedingungen der jeweiligen Staaten spielten keineswegs die Hauptrolle, sondern der Gesamtzustand des Herrschaftsgebietes wurde geprüft und erfasst. Der Vergleich betraf sowohl Gegenwart als auch Vergangenheit, denn es sollte zeitlich ungebunden alles erkannt werden, was die „gesunde Vernunft“ verlangt, um den ,gemeinen Nutzen des Staats“ zu ermitteln und zu verwirklichen. Die europäischen Staaten traten zunehmend in einen national bestimmten Konkurrenzkampf, der es erforderlich machte, die eigenen „Interessen“ gegenüber denen der anderen Staaten rational festzulegen. Die typisch aufklärerische Begründung dafür lautete: „Solchergestalt hat jedermann ... die Angelegenheiten seines Vaterlandes mit auswärtigen Mächten zu besorgen, ... Sind diese Kenntnisse ... aufgeheitert worden, so kriegen sie dadurch desto mehr Licht, und er wird die Staatsverfassung fremder Völker desto besser einsehen, je mehr er durch die Käntniß so er von seinem Vaterlande besitzet, ein Vergleichsmittel erhält, vermittelst dessen er seine Begriffe klar machen, und seinem Gedächtnisse zu Hülfe kommen kann."130

Gottfried Achenwall, der Begründer der Statistik in Göttingen, forderte ganz in diesem Sinne, die „Vergleichung des einen Staats mit allen übrigen, und kann folglich ohne vorgängige Kenntniß der übrigen Staaten nicht begriffen werden. "131

Das entsprach der Forderung des Göttinger Staatsrechtlers Johann Stephan Pütter, der mit Achenwall eng zusammen gearbeitet hatte, ,,andere Staaten mit dem seinigen in

126 BOSE, A. Introductio generalis in notitiam rerumpublicarum orbis universi ... Jenae, 1676, S. 4: ... cognoscere, quam ex inspectione accuratiore et comparatione variorum Imperiorum, quae Notitia nostra describit. OTTO, E. Notitia praecipuarum Europae rerumpublicarum. Ed. 5. Ienae, 1749; SCHMAUSS, J. J. Einleitung zu der Staats-Wissenschaft und Erleuterung ... Erster Theil. Die Historie der Balance von Europa, ... Leipzig, 1741; SCHLÖZER, A. L. Theorie der Statistik. Nebst Ideen über das Studium der Politik überhaupt. Göttingen, 1804; RASSEM, M. - STAGL, J. (hrsg.). Statistik und Staatsbeschreibung in der Neuzeit vornehmlich im 16.-18. Jahrhundert. Paderborn et al.: Schöningh, 1980; MOHNHAUPT, H. „Europa“ und ,ius publicum“ im 17. und 18. Jahrhundert. In: Aspekte europäischer Rechtsgeschichte. Festgabe für Helmut Coing zum 70. Geburtstag (Ius Commune, Sonderhefte 17). Frankfurt am Main: V. Klostermann, 1982, S. 219-224.

127 OTTO, op. cit., S. 6 (§ XI).

128 SCHMAUSS, op. cit., Vorrede.

129 ACHENWALL, G. Staatsverfassung der heutigen vornehmsten Europäischen Reiche und Völker im Grundrisse. 5. Aufl. Göttingen, 1768, S. 13.

130 DU BUAT-NANÇAY, L. G. Geschichte der alten Staatsverfassung in Frankreich, Teutschland und Italien ... Aus dem Französischen übersetzt. In vier Theilen. Bamberg - Franckfurt - Leipzig, 1763, Einleitung, S. XII ss.

131 ACHENWALL, Staatsverfassung der heutigen vornehmsten Europäischen Reiche und Völker im Grundrisse, S. 35 (§ 54). 
Vergleichung zustellen."132 Idealistisch-kosmopolitische Motive mit einer gewissen Entnationalisierung einerseits sowie utilitaristisch-politische Ziele mit starken nationalen Intentionen andererseits bestimmten diese vergleichenden Beobachtungen und Ermittlungen empirisch gewonnener Daten. Reisen, Reiseberichte und Gesandtschaftsberichte sind oft die Quellen für den empirischen Befund. Der enge Zusammenhang, in dem die europäische Staatenwelt stand, wurde erkannt und immer wieder thematisiert. Christian Gottfried Hoffmann, Professor in Frankfurt an der Oder, betonte 1720 die „,connexion“, in der ein ,jedweder Staat mit den anderen stehet." 133 Die synoptisch angelegten Darstellungen umfassen einerseits juristische Tatbestände - nämlich das ,ius publicum “ - und andererseits allgemeine natürliche Zustände der Staaten, wie sie Montesquieu 1748 zur Grundlage seines „De 1'Esprit des Lois“ gemacht hatte, und die beide die Vergleichungen tragen und ermöglichen. Diese „,comparatio diversorum civitatum“ betraf in der Regel die „notitia praecipuarum Europae civitatum“, wobei der in die Vergleichung einbezogene Kreis der Staaten unterschiedlich weit gezogen wurde. Zeitlich sollte der Vergleich synchron und diachron durchgeführt werden und zeigt damit auch ein europäisch geprägtes Bildungsprogramm: In singulis tempus praeteritum, praesens et futurum considerari debet, ut totidem prudentiae partes, memoria, intelligentia et providentia instruantur. ${ }^{134}$

Diese Vergleichung überspringt bei manchen Autoren zeitlich und territorial europäische Grenzen vor allem in Bezug auf das „Commercien-Wesen“. Der Göttinger Historiker Gatterer verfolgte 1773 sogar den Plan einer „Welt-Statistik“ als „Inbegriff der Individual- und Spezial-Statistiken aller in einem gegebenen Zeitalter bekannten Staaten“.135 Das entsprach der Aufteilung in das „,intérêt général d'une nation“ und in die „divers intérêts particuliers“. ${ }^{136}$ Vor allem Johann Heinrich Gottlieb von Justi hat 1762 in seinen „Vergleichungen der Europäischen mit den Asiatischen Regierungen“ den Eurozentrismus scharf attackiert, der „,himmelschreiende Ungerechtigkeiten begeht“ ${ }^{\prime 137}$ Er erklärt sein Vergleichsziel so: „Ich will ... die hohe Einbildung ... mäßigen, die wir Europäer von uns selbst haben, Ich mache hier den Anfang, indem ich die Beschaffenheit der Europäischen Regierungen mit dem Regierungszustande der Asiatischen und anderer vermeintlich Barbarischen Völker in Vergleichung stelle." 138

Justi kündigte zugleich an, zu einem späteren Zeitpunkt auch noch die Sitten und Religionen der Europäer und Asiaten ,in Vergleichung (zu) ziehen“. 139 So lautete auch die Forderung des Göttinger Staatsrechtlers Johann Stephan Pütter, jenseits aller Ländergrenzen

132 PÜTTER, J. St. Neuer Versuch einer Juristischen Encyclopädie und Methodologie. Göttingen, 1767, S. 29 (§ 52).

133 HOFFMANN, Chr. G. Entwurf einer Einleitung zu dem erkänntniß des gegenwärtigen Zustandes von Europa, worinnen von denen hierzu nöthigen Wissenschaften überhaupt geurtheilet ... Leipzig, 1720, S. 4 ss.; ähnlich ACHENWALL, G. Geschichte der allgemeinen Europäischen Staatshändel des vorigen und jetzigen Jahrhunderts im Grundrisse, neue Auflage. Wien, 1780, Vorrede.

134 OTTO, op. cit., S. 4 (§ VIII).

135 Cf. SCHLÖZER, op. cit., S. 87.

136 AZUNI, D.-A. Systême universel de principes du droit maritime de l'Europe. Traduit de 1'italien par J. M. Digeon. Tome I. Paris, 1797, VI, S. 16.

137 VON JUSTI, J. H. G. Vergleichungen der Europäischen mit den Asiatischen und andern vermeintlich Barbarischen Regierungen, in drey Büchern verfasset. Berlin - Stettin - Leipzig, 1762, Vorrede (s.p.).

138 VON JUSTI, op. cit., Vorrede s.p. (S. IV ss.)

139 Ibidem, Vorrede s.p. (p. V). 
„die Kenntniß eines jeden Staatsrechts“ zu nutzen und „mehr andere Staaten mit dem seinigen in Vergleichung (zu) stellen." ${ }^{140}$ Der Blick sollte auf ,alle Rechte jeder Völker und Zeiten, soweit nur glaubwürdige Nachrichten reichen“, gerichtet werden. ${ }^{141} \mathrm{Im}$ gleichen Sinne hatte Feuerbach zu Beginn des 19. Jahrhunderts - wie auch später 1876 der Richter Albert Hermann Post in seinen „Prolegomena zu einer allgemeinen vergleichenden Rechtswissenschaft“142 - eine „Universaljurisprudenz“ gefordert, für die alle Daten zur „geschichtlichen Erforschung“ der Rechtssysteme von gleichrangiger Bedeutung sein sollten: „Kein Volk, kein Stamm sei uns in dieser Hinsicht zu gering oder zu verächtlich."143 Von hier führt der Weg zur etwas nebulosen „Universalrechtsgeschichte“ Kohlers ${ }^{144}$ und direkt zur modernen Rechtsvergleichung, für die Ernst Rabel 1924 gefordert hatte: „Der Stoff des Nachdenkens muß das Recht der gesamten Erde sein, vergangenes und heutiges ...". 145

Diese „Verkettung der europäischen Völker“, wie sie Findeisen 1781 genannt hatte und die man heute wohl als ,entanglement“ bezeichnen würde, begleitete die generelle Forderung nach „Vergleichung der Wissenschaften“, um die „Völker und ihre Wissenschaften kennen zu lernen. “ 146 In der Sprache der Naturwissenschaften forderte der Göttinger Natur- und Völkerrechtler Gottfried Achenwall 1779 auch für die „Staatswissenschaften“, „eigene Erfahrungen“ zu sammeln, die „theils durch Experimente oder Versuche, theils durch Observationen oder Wahrnehmungen erlangt“" werden. ${ }^{147}$ Der „historische Beweis“ sollte neben den „mathematischen Beweis“ treten. Ein Mittel zu diesem Zweck war die Vergleichung, wie sie im Zitat des Physikers Georg Christoph Lichtenberg zum Ausdruck kommt. ${ }^{148}$

\section{Gesetzgebungsvergleich als universale Aufgabe}

Im Bereich der öffentlichen Sphäre des Rechts gewinnt die Gesetzgebung in Theorie und Praxis im Laufe des 18. und 19. Jahrhunderts eine immer größere Bedeutung. Aus der „notitia rerum publicarum" folgte nun die „,collatio et notitia exterarum legum“ - der

140 PÜTTER, Neuer Versuch einer Juristischen Encyclopädie und Methodologie, S. 29 (§ 52).

141 Ibidem, S. $23(\S 43)$.

142 Cf. KIESOW, op. cit., S. 71.

143 FEUERBACH, P. J. A. Idee und Nothwendigkeit einer Universaljurisprudenz. Naturrecht, Rechtsphilosophie, allgemeine Rechtswissenschaft. In: FEUERBACH, L (hrsg.). Anselm Ritter von Feuerbachs Biographischer Nachlaß II. 2. Ausgabe. Leipzig, 1853. Repr. Aalen: Scientia Verlag, 1973, S. 399. Solche Vergleichsforderungen wurden im 19. Jahrhundert auch an die Nationalökonomie gerichtet; cf. ROSCHER, W. Grundriß zu Vorlesungen über die Staatswirthschaft. Nach geschichtlicher Methode. Göttingen: Dieterich, 1843, S. IV: ,....alle Völker, deren wir irgend habhaft werden können, in wirtschaftlicher Hinsicht miteinander zu vergleichen."

KOHLER, J. Rechtsphilosophie und Universalrechtsgeschichte. In: VON HOLTZENDORFF, F. - KOHLER, J. (hrsg.). Encyklopädie der Rechtswissenschaft I. 7. ed. München et al.: Duncker und Humblot J. Guttentag, 1915, S. 1, 14 ss.

145 RABEL, E. Aufgabe und Notwendigkeit der Rechtsvergleichung. Rheinische Zeitschrift für Zivil- und Prozessrecht, 1924, 13, S. 279, 283.

146 Vergleichung der Wissenschaften und Künste alter und neuer Zeiten. Nach Findeisens Urtheile über die Grundsätze der alten Welt. Ein zweiter Versuch zur weitern Untersuchung. Hamburg, 1781, S. 66.

147 ACHENWALL, G. Die Staatsklugheit nach ihren ersten Grundsätzen entworfen. 4. Ausgabe. Göttingen, 1779, Vorrede, S. XXXI.

148 Cf. oben S. 39 mit n. 60. 
Gesetzesvergleich, wie z.B. Barbeyrac diese „collatio“ bezeichnet hatte. ${ }^{149}$ Das Staatsrecht war dafür ein Vorbild. Der Privatrechtler Gustav Hugo suchte 1798, die „,verschiedenen positiven Rechte“ nach „Ursachen und Wirkungen miteinander zu vergleichen“: „Es muß dies, was bisher nur im Staatsrechte geschah, hauptsächlich im Privatrechte geschehen. "150 Unter den konkurrierenden Rechtsquellen war die Gesetzgebung im Rang vor der Rechtswissenschaft und Rechtsprechung zum führenden Steuerungsinstrument aufgestiegen, was für die Gesetzgebungspraxis eine vergleichende Beobachtung vorliegender Gesetzgebungsprodukte notwendig machte. Der Beobachtungsraum war ganz Europa in seiner gesamten historischen Dimension. Die Vergleichung kulminierte in der Kodifikationsepoche des 18./19. Jahrhunderts. ${ }^{151}$ Der Aufklärer und Jurist Christian Thomasius hatte bereits 1701 die ,comparation und Gegeneinanderhaltung derer unterschiedenen hier und da gegebenen Gesetze... “ als Erkenntnismittel gefordert. ${ }^{152} 1702$ suchte er in diesem Sinne das beste Gesetz durch diachrone Vergleichung aller vorliegenden antiken Gesetze „,comparative in relatione ad Leges aliorum populorum “ zu ermitteln. ${ }^{153}$ Unter einem ethischmoralischem Gesichtspunkt forschte er nach dem Gesetz, das am besten die verdorbenen Sitten seiner Zeit zu verbessern versprach. Er fand es im Gesetz von Moses: ... lex Mosaica in comparatione ad alias leges omnes fuerit perfectissima. ${ }^{154}$ Das Mosaische Gesetz wieder einzuführen, hielt auch Thomasius nicht für realistisch, denn es sei wohl einem christlichen Regenten jetzt nicht zuzumuten, ein jüdisches Gesetz in einer christlichen Welt zu reaktivieren. Aber als ein Erkenntniswert besaßen selbst die antiken Gesetze ,aller Völker und Zeiten“ - wie Pütter und Feuerbach erklärt hatten ${ }^{155}$ - einen hohen Nutzen für das aufgeklärte und aufklärende 18. Jahrhundert, um ganz im Geiste Montesquieus historische, anthropologische, geographische und klimatische Bedingungen für eine optimale Einrichtung von Staat und Gesellschaft durch Recht aufdecken zu können. Diese Strategie verfolgt in einer zunehmend globalisierten Welt auch die moderne Rechtsvergleichung, die noch den philosophischen Geist des 18. Jahrhunderts atmet.

\footnotetext{
149 DE BARBEYRAC, J. Oratio de coniugendis iurisprudentiae et historarum studiis. In: BUDER, Chr. G. De ratione et methodo studiorum iuris ... Jenae, 1724, S. 200.

150 So HUGO, G. Lehrbuch des Naturrechts, als einer Philosophie des positiven Rechts. Berlin, 1798, Vorrede und S. 10.

151 Cf. die Übersicht zum Gesetzgebungsvergleich in dieser Epoche bei MOHNHAUPT, Vergleichende Beobachtung von Staat, Gesellschaft und Recht im 18. Jahrhundert als Vorform der modernen Rechtsvergleichung, S. 17-24.

152 THOMASIUS, Chr. Allerhand bißher publicirte kleine teutsche Schriften. Halle, 1701, Vorrede s.p. (S. VIII).

153 THOMASIUS, Chr. Lectiones de prudentia legislatoria. STOLLE, G. (hrsg.). Francofurti - Lipsiae, 1740, S. 95 (Cap. IX).

154 Ibidem, S. 122 (Cap. X, § 2).

155 Cf. oben S. 48-49 mit n. 141-143.
} 